F. James from CERN reports on recent work showing that newer types of random number generators are not as reliable as was once supposed.

\title{
Good Generators Give Wrong Answers
}
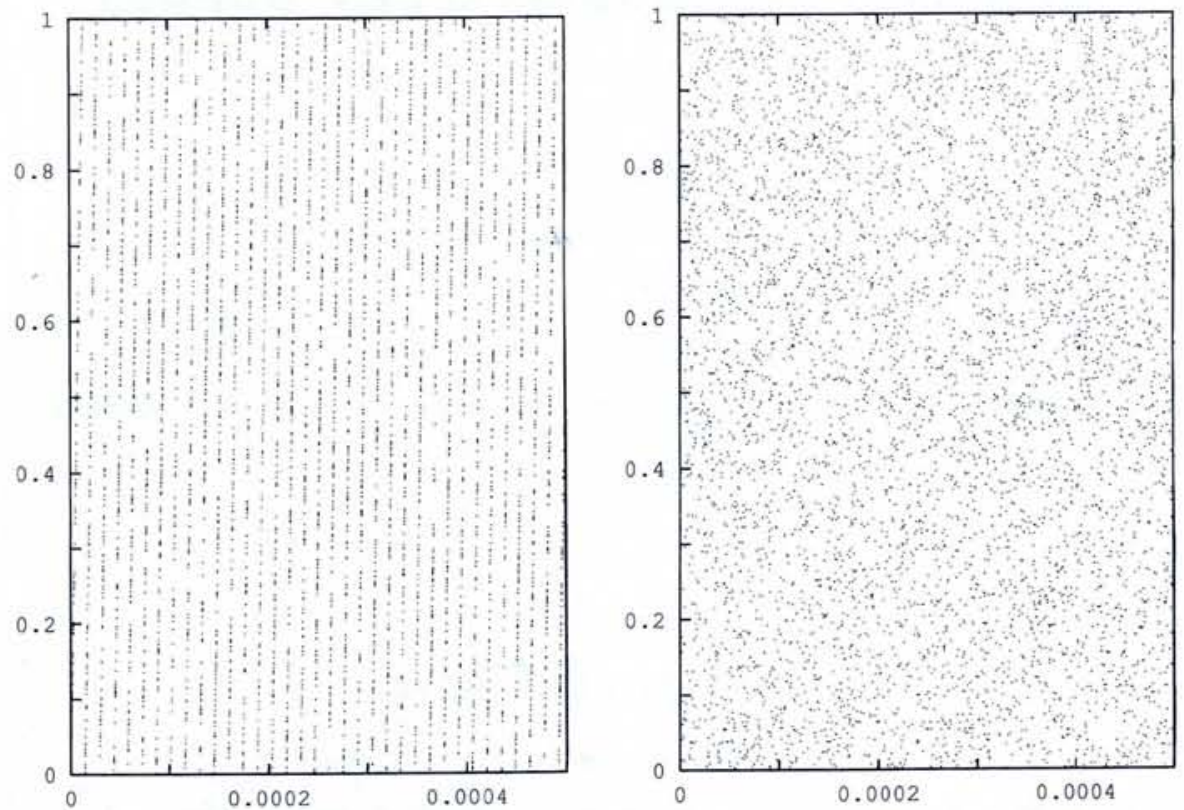

Illustration of the known defect in congruential random numbers. left) Pairs of successive numbers are plotted as $(x, y)$ coordinates in the unit square, and the tiny strip with $x<0.0005$ is magnified to show the unwanted band structure (left figure). The figure on the right shows how the structure disappears when one of the newer generators (Marsaglia's "subtract with borrow") is used. For each figure, ten million points were generated and five thousand are displayed. It is not yet known how to illustrate the defect present in the newer methods.

It has long been known that subtle effects in pseudorandom number generators can lead to incorrect results in Monte Carlo calculations. The most commonly used generators have been of the type known as linear congruential; their main defect was discovered in the early 1960's and was explained in a landmark paper by G. Marsaglia in 1968 [Proc. Nat. Acad. Sci. 61 (1968) 25]. Further research has led to a rather complete understanding of this defect and its effects can be made arbitrarily small. More recently, new classes of generators have been developed, some by Marsaglia himself, which do not have this defect at all and perform much bet- ter in statistical testing, as well as having other desirable properties such as portability and an extremely long period. These generators are described in a recent review article by F. James in Computer Physics Communications 60 (1990) 329. Until a month or two ago, it was believed that these new generators were better in every way than the congruential ones, although, as reported in the abovementioned review, "the only precise theoretical understanding is the knowledge of the period".

Now Alan Ferrenberg and collaborators from the University of Georgia and IBM Kingston report in Physical Review Letters 69
(1992) 3382 that for certain Monte Carlo Ising lattice calculations where the exact answer is known, two of the new generators (shiftregister with lag 250 and Marsaglia's subtract-with-carry) produce significantly wrong answers, whereas a congruential generator with known defects gives the right answers.

The authors suggest some recipes which improve the accuracy of results when the supposedly good generators are used, but the real nature of the defect is not yet known and may take some time to understand. In the meantime, there is still no mathematical proof that any pseudorandom number generator is random enough for any calculation, so confidence in results was based mainly on the absence of counterexamples. As computers become more powerful and Monte Carlo methods more widespread, the issue of the quality of random numbers is becoming a critical one.

If physics is your business...

\section{EXHYSICS WORLD O}

20-22 April 1993

The Brighton Centre, UK

..then you must be in Brighton in April

\section{The Exhibition}

- The largest and most important exhibition of physics-related products and services ever held in the UK

- Over 100 companies showing the very latest technologies

- Linked to the IOP Annual Congress

- Free entrance with pre-registration

For your free visitor ticket call Geraldine Pounsford on +44-272-29 7481

\section{The Congress}

- Over 1000 delegates places available, with international speakers discussing the latest technologies

- Four major conferences (spectroscopy; plasma physics; vacuum, plasma and surface technology; higher education) with 12 parallel one-day meetings For further Congress details call Lucy Bell on +44-71-235 6111

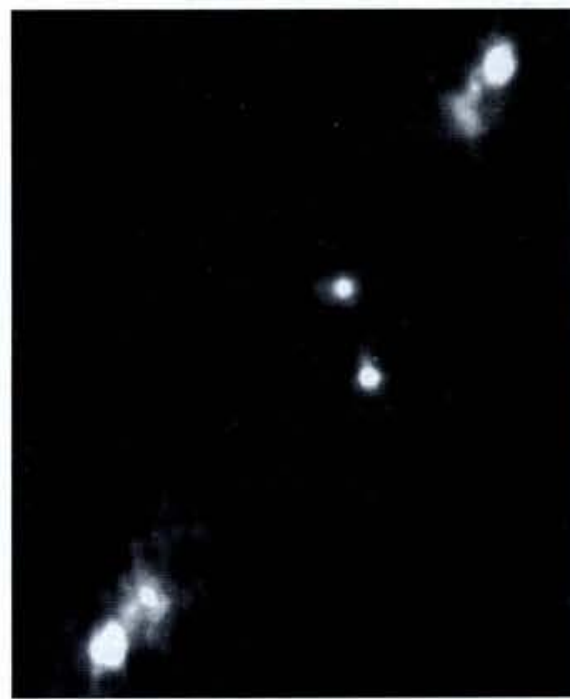

This Hubble Space Telescope photograph made in one of the first long (six hour) exposures with the Wide Field Camera shows a pair of L-shaped images with a striking mirror symmetry. They are thought to arise from a very distant galaxy seen through the AC114 cluster of foreground galaxies. The gravity of the galaxy cluster acts as a natural magnifying glass, bending and focussing the light of the more distant source into several images, each of which is apparently larger and brighter than would otherwise be the case. The two bright objects in the centre are thought to be unrelated galaxies in the foreground cluster.

While it was known from ground-based images that the cluster could act as a gravitational lens, the Hubble image being of a much higher quality allows the bending power of the lens to be measured accurately to give a precise determination of the distribution of matter in the cluster. AC114 probably has a dense, massive core as the L-shaped images are unusually far apart for a lensed system.

By fitting observed images to images obtained by modelling the gravitational field of the lens, astronomers will be able to refine the properties of the lens to the point that the AC114 gravitational lens cluster (and eventually other clusters) can be used to observe distant objects that are too faint for conventional techniques. 